Document downloaded from:

http://hdl.handle.net/10251/62240

This paper must be cited as:

Paolini, C.; Letizia, R.; Napoli, F.; Ni, Q.; Rennie, A.; André, F.; Pham, K.... (2015). Horizon 2020 TWEETHER project for W-band high data rate wireless communications. En Vacuum Electronics Conference (IVEC), 2015 IEEE International. Institute of Electrical and Electronics Engineers (IEEE). 1-2.

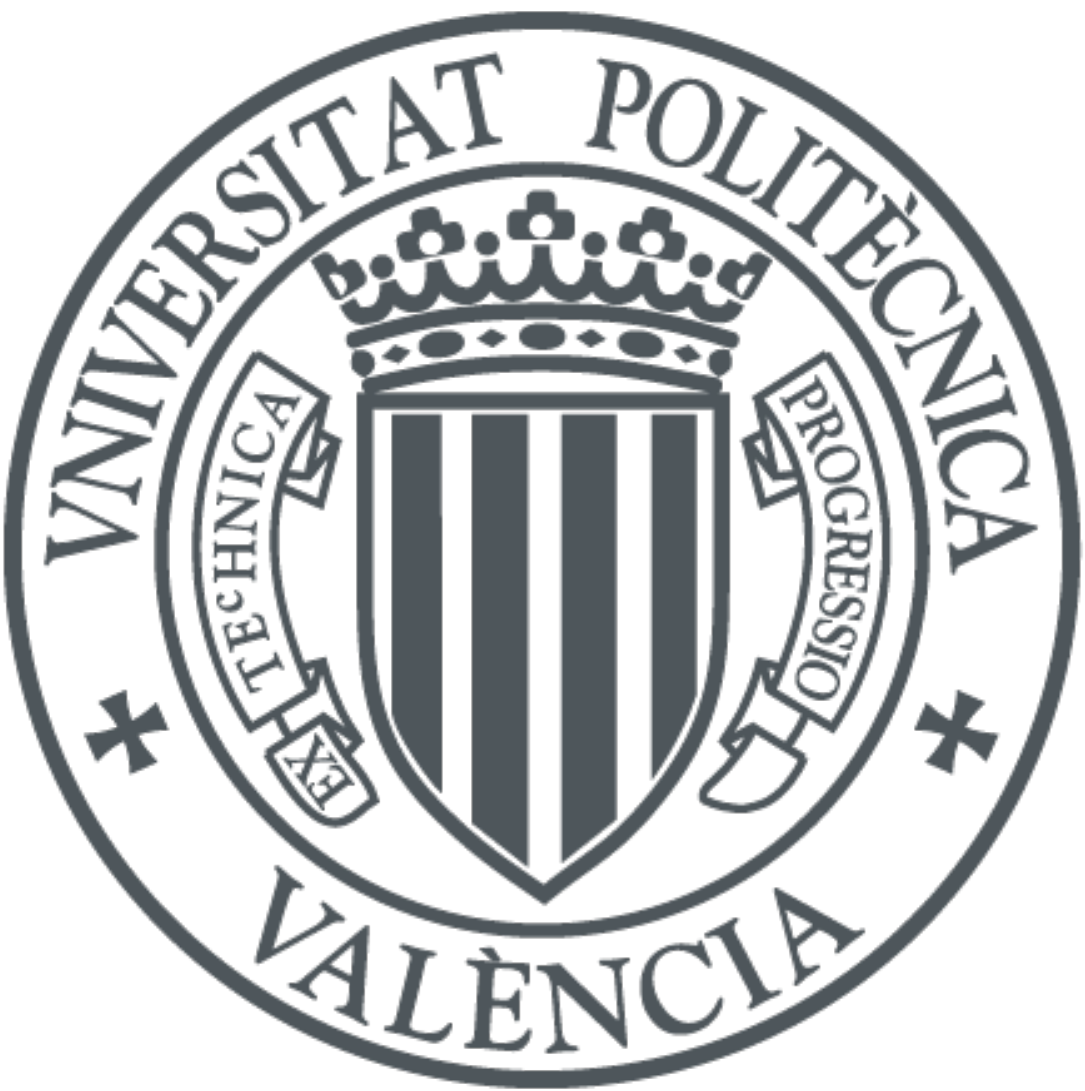

The final publication is available at

http://ieeexplore.ieee.org/xpl/mostRecentlssue.jsp?punumber=7209113

Copyright Institute of Electrical and Electronics Engineers (IEEE)

Additional Information 


\section{Horizon 2020 TWEETHER project for W-band high data rate communications}

\author{
Claudio Paoloni \\ Rosa Letizia \\ Francesco Napoli \\ Qiang Ni \\ Allan Rennie \\ Lancaster University \\ Lancaster, UK, LA1 4YR HFSE
Germany \\ Ralph Zimmerman
}

\author{
Frédéric André \\ Kim Pham \\ Thales Electron Devices \\ SAS \\ Vélizy-Villacoublay, France
}

\author{
Viktor Krozer \\ Goethe University of \\ Frankfurt \\ Frankfurt, Germany
}

\author{
François Magne \\ Ioan Burciu \\ Bluwan \\ Le Pecq, France
}

\author{
Marc Rocchi \\ Marc Marilier \\ OMMIC \\ Limeil-Brevannes, France
}

\author{
Antonio Ramirez \\ Fibernova \\ Valencia, Spain
}

\author{
Ruth Vilar \\ Universitat Politecnica de \\ Valencia \\ Valencia, Spain
}

\begin{abstract}
The outstanding demand of high data rate in wireless network is exceeding the actual capacity making the microwave region of the spectrum inadequate for the future needs. The millimeter wave region has been demonstrated suitable for multi-gigabit transmission. Unfortunately, technological issues still prevent its adequate exploitation.

The Horizon 2020 TWEETHER project "Traveling wave tube for $W$-band wireless networks with high data rate distribution, spectrum and energy efficiency" aims to respond to this challenge. A novel $W$-band traveling wave tube will be the core of the system.
\end{abstract}

Keywords: traveling wave tube; W-band; wireless communications; systems

\section{Introduction}

Wireless communications are becoming a major vehicle of exchange of information of the modern age. The constant and fast growth of mobile devices, such as smartphones and tablets, the diffusion of laptops and the availability of new video formats at high resolution are pushing the available technology at its limits. The frequencies of the new $4 \mathrm{G}$ and future $5 \mathrm{G}$ networks it is not expected to exceed a few GHz. The use of pico and nano cells to overcome the power limitation at those frequencies is an effective approach. However, the distribution of high data rate in many suburban and residential areas is predicted to be prevented by the difficulty of installing fibers to properly feed the numerous hubs.

In principle, the millimeter wave portion of the spectrum, allocating multi-gigahertz signal, offers the solution to distribute multi gigabit data rate $[1,2]$. Different communications links were presented with relevant performance in terms of data rate. Solid-state electronics at millimeter wave and in particular at $\mathrm{W}$-band is progressing fast to provide the adequate chipset for a proper signal processing [3]. Unfortunately, at millimeter wave frequencies, the lack of powerful amplifiers, the high costs of electronics and the high atmospheric attenuation (in particular in rain condition) have prevented so far the exploitation of this frequency band. GaN based devices provide output power at 1 Watt level [4], but this figure is still far from that required for a proper operation.

Horizon 2020 TWEETHER project "Traveling wave tube for W-band wireless networks with high data rate distribution, spectrum and energy efficiency" aims to design, construct and test a transceiver at W-band to distribute high speed data rate, both for access and backhaul based on a novel W-band traveling wave tube.

\section{TWEETHER project aims}

The link budget at millimeter wave frequencies is highly affected by atmospheric attenuation, particularly critical in raining condition. The high transmission power required to satisfy the link specification exceeds the level achievable by solid-state devices. The recent progress in vacuum electron devices offers new opportunities to create a new generation of wireless network for high data rate to satisfy the huge increase of data traffic and the widest internet distribution for mobile and fixed connection networks. The enabling device of the new $\mathrm{W}$ band transceiver (Fig.1) will be a novel wide band W-band traveling wave tube (TWT). 


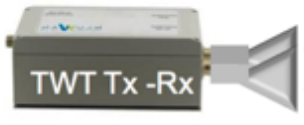

Hub

(Tx W-band TWT)

(Rx W-Band MMIC)

Figure 1. Transceiver concept (ODU Outdoor Unit)

A traveling wave tube is the only device capable to provide the level output power to distribute millimeter wave frequency signals in a wide area up to a useful distance to assure convenient user coverage. Typically, TWTs are based on helix slow wave structures (SWSs). At the increase of the frequency the diameter of the helix decreases substantially, posing serious fabrication limits (e.g., at W-band, a helix should have a diameter in the order of 100 microns). It has been demonstrated that the use of novel topologies of SWSs realized by high accuracy microfabrication techniques can make feasible a new family of vacuum electron tube amplifiers in millimeter wave and sub- $\mathrm{THz}$ range [4 - 6].

The W-band TWT will be integrated within the transceiver. The transceiver includes a novel W- band chipset based on Monolithic Microwave Integrated Circuit (MMIC), a high performance synthesizer. Different antennas for different targets will be considered.

A compact receiver based on W-band MMIC will be designed and realized.

The full TWEETHER transceiver will be tested in an outdoor test field based on an urban environment.

\section{Conclusions}

A large European consortium led by Lancaster University is working in the frame of the EU Horizon 2020 TWEETHER project to demonstrate the strong impact of using different technology at the state of the art, such as the vacuum electronics and millimeter wave integrated circuits as solution for the digital divide that affects a large portion of the population living in sub-urban and residential areas.

\section{Acknowledgements}

The project has received funding from the European Union's Horizon 2020 research and innovation programme under grant agreement No 644678

\section{References}

1. J. Wells, "Faster than fiber: The future of multi-G/s wireless," in Microwave Magazine, vol. 10, no. 3, pp. 104-112, May 2009.

2. J. Takeuchi, et. al. "10-Gbit/s Bi-directional wireless data transmission system using 120-GHz-band orthomode transducers," in 2012 IEEE Radio and Wireless Symposium, 2012, pp. 63-66.

3. Zaknoune, M., Okada, E., Mairiaux, E., Roelens, Y., Ducatteau, D., Frijlink, P., Rocchi, M., Maher, H., "0.2- InP/GaAsSb DHBT Power Performance With 10 and 25\% PAE at $94 \mathrm{GHz}$," IEEE Electron Device Letters, vol.35, no.3, pp.321,323, March 2014.

4. Micovic, M., Kurdoghlian, A., Shinohara, K., Milosavljevic, I., Burnham, S.D., Hu, M., Corrion, A.L., Wong, W.S., Schmitz, A., Hashimoto, P.B., Willadsen, P.J., Chow, D.H., Fung, A., Lin, R.H., Samoska, L., Kangaslahti, P.P., Lambrigtsen, B.H., Goldsmith, P.F., "W-Band GaN MMIC with 842 mW output power at $88 \mathrm{GHz}$," Microwave Symposium Digest (MTT), 2010 IEEE MTT-S International , vol., no., pp.237,239, 23-28 May 2010

5. M. Mineo and C. Paoloni, "Double Corrugated Rectangular Waveguide Slow-wave Structure for $\mathrm{THz}$ Vacuum Devices", IEEE Trans. on Electron Devices. Vol.57, N.11, pp.3169-3175, November 2010.

6. C. Paoloni, A. Di Carlo, F. Bouamrane, T. Bouvet, A. J. Durand, M. Kotiranta, V. Krozer, S. Megtert, M. Mineo, and V. Zhurbenko, "Design and Realization Aspects of 1-THz Cascade Backward Wave Amplifier Based on Double Corrugated Waveguide," IEEE Transactions on Electron Devices, vol. 60, no. 3, pp. 1236-1243, Mar. 2013.

7. C. Paoloni and M. Mineo, "Double Corrugated Waveguide for G-Band Traveling Wave Tubes," IEEE Transactions on Electron Devices, vol.61, pp.4259 4263, Dec. 2014, 